\title{
On the Permeability of Fractal Tube Bundles
}

\section{Journal Article}

\section{Author(s):}

Zinovik, I.; Poulikakos, D.

Publication date:

2012-09

Permanent link:

https://doi.org/10.3929/ethz-b-000054583

Rights / license:

In Copyright - Non-Commercial Use Permitted

Originally published in:

Transport in Porous Media 94(3), https://doi.org/10.1007/s11242-012-0022-0 


\title{
On the Permeability of Fractal Tube Bundles
}

\author{
I. Zinovik · D. Poulikakos
}

Received: 13 April 2011 / Accepted: 8 May 2012 / Published online: 12 June 2012

(C) Springer Science+Business Media B.V. 2012

\begin{abstract}
The permeability of a porous medium is strongly affected by its local geometry and connectivity, the size distribution of the solid inclusions, and the pores available for flow. Since direct measurements of the permeability are time consuming and require experiments that are not always possible, the reliable theoretical assessment of the permeability based on the medium structural characteristics alone is of importance. When the porosity approaches unity, the permeability-porosity relationships represented by the Kozeny-Carman equations and Archie's law predict that permeability tends to infinity and thus they yield unrealistic results if specific area of the porous media does not tend to zero. The aim of this article is the evaluation of the relationships between porosity and permeability for a set of fractal models with porosity approaching unity and a finite permeability. It is shown that the tube bundles generated by finite iterations of the corresponding geometric fractals can be used to model porous media where the permeability-porosity relationships are derived analytically. Several examples of the tube bundles are constructed, and the relevance of the derived permeability-porosity relationships is discussed in connection with the permeability measurements of highly porous metal foams reported in the literature.
\end{abstract}

Keywords Permeability - Geometric fractals - Metal foams

\section{List of Symbols}

$L \quad$ Length of tube bundle, $\mathrm{m}$

$\Delta P \quad$ Pressure drop across tube bundle, $\mathrm{Pa}$

$d \quad$ Tube diameter, $\mathrm{m}$

$q \quad$ Tube flow rate, $\mathrm{m}^{3} / \mathrm{s}$

$\mu \quad$ Dynamic viscosity, Pas

\footnotetext{
I. Zinovik $(\varangle) \cdot$ D. Poulikakos

Laboratory of Thermodynamics in Emerging Technologies, Department of Mechanical and Process Engineering, ETH Zurich, Zurich 8092, Switzerland

e-mail: izinovik@ethz.ch

D. Poulikakos

e-mail: dimos.poulikakos@ethz.ch
} 
$\beta \quad$ Tube flow form factor, -

$\alpha \quad=(\beta / \mu)(\Delta P / L), \mathrm{m}^{-1} \mathrm{~s}^{-1}$

$n \quad$ Number density of distribution of diameters $d$ of bundle tubes $\mathrm{m}^{-1}$

$D$ Fractal dimension, power coefficient of power law function, -

$\tau \quad=1+D,-$

$C$ Constant pre-factor of the power law function, $\mathrm{m}^{(\tau-1)}$

$N \quad$ Cumulative number distribution, -

$\varphi \quad$ Porosity, -

$Q \quad$ Total flow rate of tube bundle, $\mathrm{m}^{3} / \mathrm{s}$

$m_{k} \quad$ k-moment of distribution density function, $n, \mathrm{~m}^{k}$

$A \quad$ Cross sectional area of tube bundle, $\mathrm{m}^{2}$

$K_{D}=\frac{\mu}{\Delta P / L} \frac{Q}{A}$; Darcy permeability, $\mathrm{m}^{2}$

$N_{k} \quad$ Number of tubes that are added into the bundle at iteration " $k$ ", -

$x=N_{k+1} / N_{k}$; Pattern (tube number) scaling factor, -

$a_{k} \quad$ Area of cross section of tube which is added at iteration $k, \mathrm{~m}^{2}$

$y=\left(a_{k} / a_{k+1}\right)^{1 / 2}$; Length scaling factor, -

$f \quad$ Subscript index denoting parameters related to fractal, -

$K_{f}=\frac{K_{\text {Df }}}{\beta A}$; Dimensionless fractal permeability, -

$K=\frac{K_{D}}{\beta A}$; Dimensionless permeability of prefractal bundle, -

p,r Fitting parameters of the Archie's law, $\mathrm{m}^{2},-$

\section{Introduction}

Fractals are proven to be a useful model of porous media with complex structure. The results of application of fractal models to analysis of flow in porous structures are covered in extensive reviews (Gimenez et al. 1997; Perfect et al. 2006; Perrier et al. 2000; Yu and Liu 2004). Such studies are often focused on the link between permeability and the fractal dimension of porous structures. A systematic study of this topic begins with the analysis given by Jacquin and Adler (1987), where a porous medium is modeled as a set of parallel solid rods with an axial Stokes, flow around the solid. The cross section of the medium is represented by a prefractal of the Sierpinski carpet shown in Fig. 1 (white squares depict the solid inclusions). An extensive series of numerical simulations of the flow is conducted for different prefractals with the porosity decreasing to zero. The results show that the permeability can be approximated by a power-law function of the porosity if the power coefficient equals $(4-\mathrm{D}) /(2-\mathrm{D})$ where $\mathrm{D}$ is the dimension of the fractal set. This power law approximation of the permeability of a fractal medium as a function of porosity can be seen as an extension of the Kozeny-Carman equation and is called Archie's law (Gimenez et al. 1997). Further investigations indicate that for low-porosity media, Archie's law exponent can either be defined as a function of the fractal dimension or serves as a fitting parameter (Henderson et al. 2010) of empirical correlations. In these equations, the permeability is inversely proportional to one minus the porosity and thus tends to infinity when the porosity approaches unity (Henderson et al. 2010; Costa 2006).

Most of the studies of fractal models of permeability are focused on flow in consolidated soils and fractured rock where the porosity of the media approaches zero. However, there exist many applications in engineering where the limit at the other end of the porosity spectrum (porosity approaching unity) is at play. To this end, in many engineering applications involving metal foams (Boomsma and Poulikakos 2001; Boomsma et al. 2003a,b), aerogels 


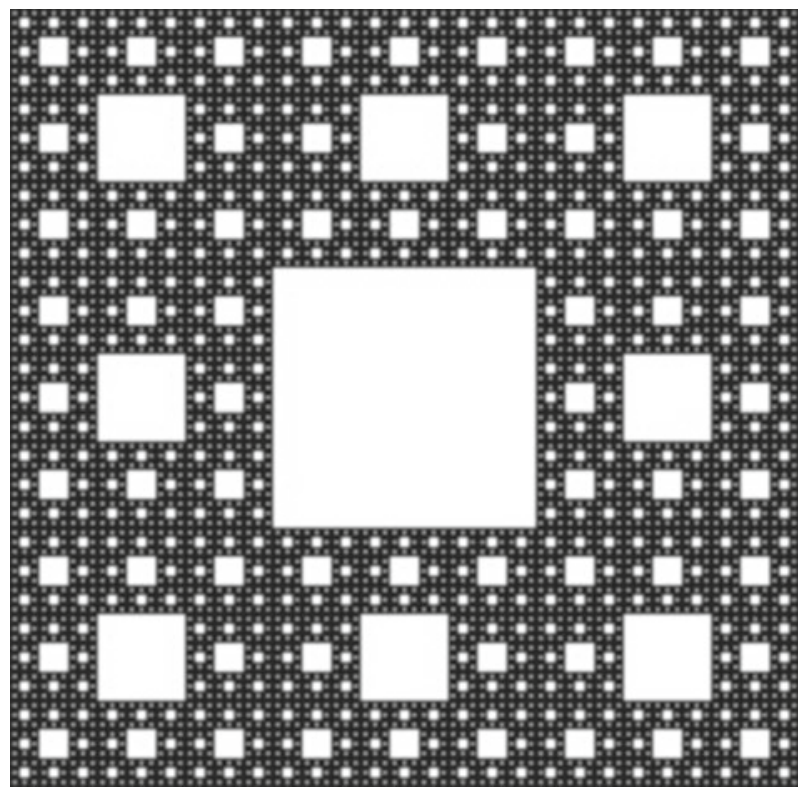

Fig. 1 Square Sierpinski carpet (as shown in wikipedia.org/wiki/List_of_fractals_by_Hausdorff_dimension)

(Pierre and Pajonk 2002; Donatti et al. 2005; Gurav et al. 2010), bone matrices (PredoiRacila et al. 2010; Fritton and Weinbaum 2009), scaffolds for tissue engineering (Deville et al. 2006; O'Brien et al. 2007; Hirata et al. 2010), bundles of carbon nanotubes (Neimark et al. 2003) filters, and catalytic reactors (Thormann et al. 2007) permeability relations for high-porosity media are necessary. Since many of the high-porosity materials also possess a complex hierarchical structure, the objective of this study is to propose fractal models for the finite permeability of porous media when the porosity tends to unity.

The model porous medium studied herein is a bundle of parallel tubes with the cross section of the bundle being specified as a two-dimensional prefractal. For example, the inversion of pore and solid spaces of the Sierpinski carpet shown in Fig. 1 can be seen as the cross section of a bundle of parallel tubes with square cross sections. If the number of iterations for the prefractal construction tends to infinity, then the porosity of the bundles tends to unity and the model porous medium is as a bundle of an infinite number of tubes with infinitely thin walls. In hydrology, the fractal models are applied to analysis of permeability of soils (Perfect et al. 2006). In the mathematical studies, similar fractals are sometimes called the sprays, and their structural properties are expressed in terms of the Riemann zeta-function (Lapidus 2010).

It is clear that the bundle of tubes with infinitely thin walls have a finite permeability since the tube walls impose a resistance on the flow. Thus, the permeability equations (Henderson et al. 2010; Costa 2006) derived for low-porosity media will fail to describe permeability of the prefractal tube bundles, since the permeability of these bundles should converge to a finite value defined by the corresponding fractal. The aim of this study is the derivation and evaluation of relationships between porosity and permeability for a set of fractal models with porosity approaching unity and a finite permeability. 


\section{Permeability of Prefractal Tube Bundle with Power Law Distribution of Tube Diameters}

First, we will derive the relation between porosity and permeability of a prefractal tube bundle making usual assumptions applicable for the analysis of the Darcy permeability of two-dimensional fractal porous media. We assume that the prefractal bundle consists of parallel tubes of a fixed length $L$ with circular cross sections, and the flow in all tubes obeys the Hagen-Poiseuille equation and is driven by the same pressure gradient $\Delta P / L$. In addition to this, we assume that the distribution of the hydraulic diameters $d$ of the bundle tubes is described by a power law.

For physical porous media, the last assumption about power-law distribution does not hold exactly but serves as an approximation of actual data. A geometric example of fractal sets that satisfy this assumption asymptotically is the Apollonian gasket. Numerical calculations (Boyd 1982; Manna and Herrmann 1991) indicate that, after a certain number of iterations, the distribution function converges to a power law with the exponent that becomes the dimension of the Apollonian fractal. The pre-factor of the power law in the case of integral Apollonian packing is numerically calculated by Fuchs and Sanden (2009).

The assumption of the Hagen-Poiseuille regime of the flow implies that the flow rate $q$ in a tube with a hydraulic diameter $d$ can be calculated as follows: $q(d)=\alpha \mathrm{d}^{4}$, where $\alpha=(\beta / \mu)(\Delta P / L), \mu$ is the dynamic viscosity of the fluid, and $\beta$ is a constant form factor for circular tubes. The number density of the distribution of the hydraulic diameters $d$ of the bundle tubes can be written as follows: $n(d)=C d^{-\tau}$, where $\mathrm{C}$ is a constant with the dimension of length in power of $\tau-1$, and the exponent $\tau$ is related to the fractal dimension $D$ via $\tau=1+D$. Using the introduced notation, the tube cumulative number distribution (the number of tubes with diameter smaller than $d$ ) $N$, the porosity $\varphi$, and the total flow rate $Q$ in the tube bundle can be written as normalized corresponding moments $m_{k}$ of the distribution density function $n(d)$ :

$$
\begin{aligned}
& N=m_{0}=\int_{d}^{d_{0}} n(\xi) d \xi=\frac{C}{D}\left(d^{-D}-d_{0}^{-D}\right) \\
& \varphi=\frac{\pi}{4 A} m_{2}=\frac{\pi}{4 A} \int_{d}^{d_{0}} n(\xi) \xi^{2} d \xi=\frac{\pi C}{4 A(2-D)}\left(d_{0}^{2-D}-d^{2-D}\right) \\
& Q=\alpha m_{4}=\int_{d}^{d_{0}} n(\xi) q(\xi) d \xi=\alpha \int_{d}^{d_{0}} n(\xi) \xi^{4} d \xi=\frac{\alpha C}{4-D}\left(d_{0}^{4-D}-d^{4-D}\right)
\end{aligned}
$$

In the Eqs. (1-3), $d_{0}$ and $d$ are the maximum and minimum hydraulic diameters in the prefractal bundle, respectively, after a certain number of iterations which generate the bundle cross-section. The cross sectional area of the bundle is denoted by $A$. If the number of iterations tends to infinity, then the minimum diameter tends to zero, while the porosity tends to unity. Thus, Eq. 2 is reduced, to the following equation:

$$
1=\frac{\pi C}{4 A(2-D)} d_{0}^{2-D},
$$

and the total flow rate in the fractal tube bundle $Q_{f}$ is obtained from the Eq. 3 when $d=0$ as follows: 


$$
Q_{f}=\frac{\alpha C}{4-D} d_{0}^{4-D}
$$

The minimum diameter $d$ can be expressed as a function of the porosity using Eq. 2. Substitution of the resulting equation into Eq. 3 and elimination of the minimum and maximum diameters from the resulting expression with the help of Eq. 4-5 leads to the following equation for the total flow rate:

$$
Q=Q_{f}\left(1-(1-\varphi)^{\frac{4-D}{2-D}}\right)
$$

The Darcy permeability $K_{D}$ of the bundle can be written using the Darcy law:

$$
K_{D}=\frac{\mu}{\Delta P / L} \frac{Q}{A}
$$

Substitution of Eq. 6 into Eq. 7 yields the final equation connecting the Darcy permeability and porosity of the prefractal porous medium:

$$
K_{D}=K_{\mathrm{Df}}\left(1-(1-\varphi)^{\frac{4-D}{2-D}}\right),
$$

where the permeability of the fractal tube bundle $K_{\mathrm{Df}}$ is

$$
K_{\mathrm{Df}}=\frac{C \beta}{(4-D) A} d_{0}^{4-D}
$$

The derivation of Eq. 8 can be repeated for an arbitrary shape of tube cross section if the hydraulic diameter $d$ is replaced by a characteristic length scale which equals the square root of the tube cross-sectional area and the form factor $\beta$ is replaced by a form factor specific to the cross section of the tubes. A collection of the corresponding form factors for various tube cross sections can be found in (Shah 1978). Note that the permeability equation of the prefractal tube bundle has the same exponent coefficient as the coefficient which is obtained in (Jacquin and Adler 1987) based on numerical simulations of the flow in a low porosity fractal porous medium. In case of the fractal tube bundle, the specific form of the exponent coefficient in the permeability-porosity Eq. 8 is due to the algebraic link between the secondand the fourth-order moments (Eqs. 2, 3) of the power-law distribution function $n(d)$.

\section{Geometric Fractals}

Since the assumption of the power law distribution of pore sizes is only an approximation, a convenient way to evaluate the permeability-porosity in actual prefractals is to utilize the geometric fractals as generating sets of the tube bundle cross section. To exemplify the derivation of the permeability-porosity relation in geometric prefractal tube bundles, we consider the Sierpinski carpet shown of Fig. 1. The following notations will be used to characterize the fractal structure: (1) the area of the bundle is denoted by $A$; (2) the number of tubes that are added into the bundle at iteration " $k$ " is denoted as $N_{k}$; (3) the area of cross section of every tube which is added at the iteration " $k$ " is $a_{k}$; (4) a pattern (tube number) scaling factor $x$ is defined as $x=N_{k+1} / N_{k}$; and (5) a length scaling factor $y$ is defined as $y=\left(a_{k} / a_{k+1}\right)^{1 / 2}$. For the square Sierpinski carpet, $N_{1}=1, a_{1}=A / 9$, and the factors $x$ and $y$ equal 8 and 3 , respectively. With these notations, the porosity of the fractal tube bundle can be written as a 
geometric series:

$$
\varphi_{f}=\frac{1}{A}\left(N_{1} a_{1}+N_{2} a_{2}+N_{3} a_{3}+\cdots\right)=\frac{1}{y^{2}}+\frac{x}{y^{4}} \sum_{k=0}^{\infty}\left(\frac{x}{y^{2}}\right)^{k}=\frac{1}{y^{2}-x}
$$

Note that since $\varphi_{f}=1$, the scaling factors have to satisfy the equation:

$$
y^{2}-x=1
$$

The equation is true if $x<y^{2}$, which is implicitly satisfied in the applied fractals, and it can be written as $\mathrm{y}^{2}-\mathrm{x}=\mathrm{N}_{1}$.

As in the previous section, we assume that the flow rates in the tubes can be calculated as $q_{k}=\alpha a_{k}^{2}$, with an adequate value of the coefficient $\alpha$. The total flow rate in the fractal tube bundle can be written as a geometric series similar to one shown in Eq. 10 but with squared denominators:

$$
Q_{f}=\sum_{k=1}^{\infty} q_{k}=\sum_{k=1}^{\infty} \alpha N_{k} a_{k}^{2}=\alpha A^{2}\left(\frac{1}{y^{4}}+\frac{x}{y^{8}} \sum_{k=0}^{\infty}\left(\frac{x}{y^{4}}\right)^{k}\right)=\alpha A^{2} \frac{1}{y^{4}-x}
$$

Substitution of Eq. 12 into Darcy's law (Eq. 7) yields the Darcy permeability coefficient for the fractal tube bundle $K_{\mathrm{Df}}$ :

$$
K_{\mathrm{Df}}=\beta A \frac{1}{y^{4}-x}
$$

To obtain the relation between permeability and porosity for the prefractal tube bundle, we apply the formula of the sum of the first $n$ terms of a geometric series to Eqs. 10 and 12:

$$
\begin{aligned}
& \varphi=\frac{1}{A}\left(N_{1} a_{1}+\cdots+N_{n} a_{n}\right)=\frac{1}{y^{2}}+\frac{x}{y^{4}} \sum_{k=0}^{n}\left(\frac{x}{y^{2}}\right)^{k}=\frac{1}{y^{2}}+\frac{x}{y^{4}} \frac{1-\left(\frac{x}{y^{2}}\right)^{n+1}}{1-\frac{x}{y^{2}}} \\
& Q=\sum_{k=1}^{n} q_{k}=\alpha A^{2}\left(\frac{1}{y^{4}}+\frac{x}{y^{8}} \sum_{k=0}^{n}\left(\frac{x}{y^{4}}\right)^{k}\right)=\alpha A^{2}\left[\frac{1}{y^{4}}+\frac{x}{y^{8}} \frac{1-\left(\frac{x}{y^{4}}\right)^{n+1}}{1-\frac{x}{y^{4}}}\right]
\end{aligned}
$$

Note that the pore size distribution function can be calculated using Eq. 14 with the coefficient $y$ set to unity and expressed as a parametric function of the power of inverse size $(1 / y)^{n}$ with the iteration number $n$ serving as the parameter of the function:

$$
N=N_{1}+\cdots+N_{n}=1+x \sum_{k=0}^{n} x^{k}=1+x \frac{1-x^{n+1}}{1-x}
$$

To simplify the notation, we introduce the dimensionless permeabilities $K_{f}$ and $K$ of the fractal and prefractal bundles, respectively:

$$
K_{f}=\frac{K_{\mathrm{Df}}}{\beta A} ; K=\frac{K_{D}}{\beta A}
$$

The denominator in the defined dimensionless permeability refers to the permeability of a single tube with the same cross section as the tube bundle, and thus the value of the dimensionless is always less than unity. In the geometric fractals, the scaling factors $x$ and $y$ are related to the fractal dimension as 


$$
x=y^{D}
$$

Equations 14 and 15 can be re-written substituting Eq. 18 for $x$ and then simplified using Eq. 11. The results yield the following equations:

$$
\begin{gathered}
\varphi=y^{-2}+y^{(D-2)}\left(1-y^{(D-2)(n+1)}\right) \\
K=y^{-4}+K_{f} y^{(D-4)}\left(1-y^{(D-4)(n+1)}\right)
\end{gathered}
$$

Eliminating the scaling factor $y^{(n+1)}$ from Eq. 19 and 20 yields the relation between permeability and porosity:

$$
K=K_{f}-K_{*}(1-\varphi)^{(4-D) /(2-D)},
$$

where

$$
K_{*}=\left[K_{f}-\frac{1}{y^{4}}\right]\left[\frac{y^{2}}{y^{2}-1}\right]^{(4-D) /(2-D)} .
$$

Equations 8 and 21 have identical exponent coefficients; thus, when the porosity tends to unity, the permeability of the geometric prefractal behaves in the same way as the permeability of a prefractal with exact power-law distribution. The distribution function $N=f\left(1 / y^{n}\right)$ of the prefractal defined by Eq. 16 can also be approximated by a power law with a high accuracy: a power-law curve with an exponent which equals the fractal dimension of the Sierpinski carpet approximates the fractal distribution function with a determination coefficient $R^{2}>0.999$. On the other hand, the functional relation between permeability and porosity of the geometrical prefractal requires not only the fractal permeability $K_{f}$ as in the case of Eq. 8 but also an additional parameter $K_{*}$ which depends on the fractal dimension.

Note that Eq. 21 does not depend on the form factor $\beta$. The permeability of prefractal bundles of the Sierpinski triangular carpet is also defined by Eq. 21 with the corresponding substitutions of the scaling factors: $x=3$, and $y=2$. The permeability-porosity relations for the geometric prefractal tube bundles can also be written in the form of Eq. 21 for the Pinwheel (Radin 1994), the Pascal triangle modulo 5 (Wolfram 1984), and the Koch snowflake prefractals (Zinovik and Poulikakos 2012). Another example of prefractal tube bundles can be constructed using the Apollonian gasket. The permeability of the Apollonian gasket can be calculated using a MATLAB script available on the website of company MathWorks which develops mathematical software (Jacquenot 2007). The shape of the permeability-porosity curve of the Apollonian prefractal resembles the curves of the other geometric prefractals and can be approximated using Eq. 21 where coefficients $K_{f}, K_{*}$, and $D$ are treated as fitting parameters (Zinovik and Poulikakos 2012). The fitted value of the coefficient $D$ was found to be 0.70 , that is, almost two times smaller than the Apollonian fractal dimension. This deviation is because fact that at the initial iterations, the size distribution function of the Apollonian gasket deviates significantly from the power law that asymptotically approximates the fractal dimension of the gasket (Manna and Herrmann 1991).

\section{Discussion}

The application of the capillary model of flow to actual three-dimensional porous media has apparent limitations. Nevertheless, this model is often used to provide a basis for discussion of possible factors influencing the flow in porous media. Since the porosity of the prefractal tube bundles tends to unity, it is useful to test if this model could serve as a tool for 


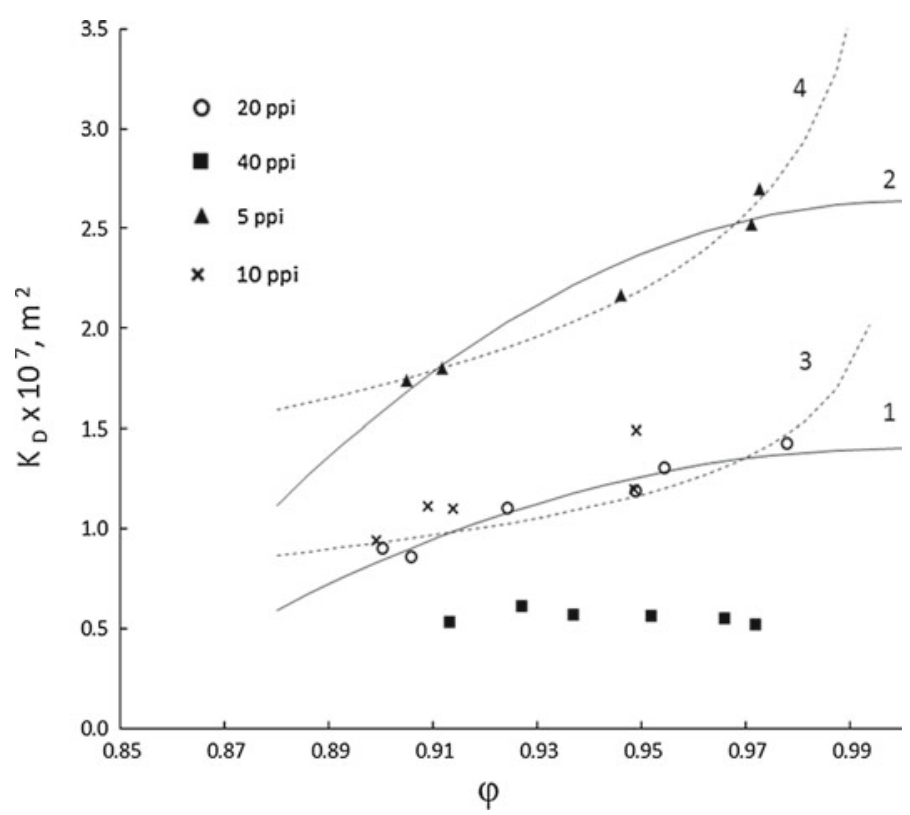

Fig. 2 Permeability of metal foams adopted from table 2 in (Bhattacharya et al. 2002); legend indicates number of pores per inch (ppi) in tested foam; 1,2 - fitting curves (20 and 5 ppi) with Eq. 21; 3,4- empirical correlation ( 20 and 5 ppi) of Rodriguez et al. 2004

approximation of permeability-porosity relationships of highly porous media. One example of a high-porosity medium is the metal foams which are often studied in connection with heat and mass transfer processes (Boomsma and Poulikakos 2001; Boomsma et al. 2003a,b).

Difficulties of estimation of the permeability of metal foams sometime lead to counterintuitive assumptions which are not supported by the experimental results. For example, semi-empirical fitting suggested in (Bhattacharya et al. 2002) for the measured permeability is based on a definition of tortuosity which predicts that the high-porosity limit of the tortuosity is not unity (see Eq. 20 in Bhattacharya et al. 2002). Such definition of tortuosity contradicts most of the experimental results reported in the literature where tortuosity is approximated as a power of the porosity.

In Fig. 2, the permeability values from Table 2 of Bhattacharya et al. 2002 are plotted as a function of porosity. The data shown in Fig. 2 does not favor the conclusion of an infinite permeability increase which would be predicted by the Archie's law in high-porosity limit over the possibility of gradual leveling of the permeability as it follows from the prefractal bundle model. Moreover, the permeability of the foam with 40 pores per inch (closed symbols 4 in Fig. 2) remains approximately the same for all the tested porosities as it would be according to the permeability-porosity formula for the prefractal bundles when the porosity approaches unity.

A possible explanation of the qualitatively similar behavior of the permeability of the metal foams shown in Fig. 2, and the prefractal tube bundles can be found in the following example: let us assume that a sample of a solid mesh is inserted into a tube with flowing fluid. If the sample is relatively small and the mesh pores are relatively large, some space will be available for flow through the sample along almost straight flow lines. This space can 
be visualized by a projection of the mesh on the plane perpendicular to the tube axis. This projection may look like a cross section of a tube bundle composed of the tubes with different hydraulic diameters. If the space available for flow along straight or slightly curved flow lines has size distribution function similar to the prefractal bundles, then the permeability-porosity relationships of such foams should be similar to the permeability formulas derived herein for the prefractal bundles.

The permeability-porosity relation defined by Eq. 21 was used as a fitting equation for approximation of permeability data for 5, 10 and 20 ppi, foam which is shown in Fig. 2. These data were also fitted by the least square method utilizing the Archie's law formula $K=p \varphi^{r} /(1-\varphi)$ for low-porosity fractals proposed in (Costa 2006) and an empirical correlation $K=p \varphi^{r+1} /(1-\varphi)^{r}$ (Rodriguez et al. 2004) developed for chaotically oriented fibers where $\mathrm{m}$ and $\mathrm{n}$ are fitting parameters. In all cases but one (dashed line in Fig. 2), the prefractal tube bundle formula provided a better fit, i.e., smaller root mean square deviation from the data. The regression coefficients are shown in Appendix.

Since the number of data points in Fig. 2 is limited, applicability of Eq. 21 as an accurate fitting formula needs further experimental investigations. Nevertheless, it is clear that fitting permeability with the Archie's law will be less accurate than with Eq. 21 if the permeability in high-porosity limit becomes almost constant as it is for the foam with 40 ppi in Fig. 2.

Derivation of the permeability equations of prefractal tube bundles may also provide an insight on when permeability curves of Eq. 8 or Eq. 21 should serve as a better approximation than the Archie's law. For the derivation of Eqs. 8 an 21, it was assumed that the prefractal bundles are constructed by iterative addition of the tubes with gradually decreasing diameters. All the steps of the derivation of these permeability equations can also be repeated assuming that the iterations add the tubes with gradually increasing diameters. For example the lower limit of the integral in Eq. 2 in this case will be zero and the upper limit will denote a maximum tube diameter which increases at every iteration. The permeability equations Eqs. 8 and 21 will then take form $K_{D}=K_{\mathrm{Df}} \varphi^{\frac{4-D}{2-D}}$ which is asymptotically equivalent to the Archie's law when the porosity tends to zero. Thus, one may conclude that the Archie's law provides a better approximation if the permeability of a porous medium increases due to addition of the pores with gradually increasing diameters, while fitting curves of Eq. 21 could be used if increase of porosity of the medium is caused by rise of the pore fractions with consequently decreasing diameters.

\section{Conclusion}

Prefractal tube bundles generated by finite iterations of the corresponding geometric fractals can be used as a model porous medium where permeability-porosity relationships are derived analytically as explicit algebraic equations. Unlike the equations of the KozenyCarman and Archie's laws, the permeability-porosity equations of the prefractals predict a finite permeability when the porosity approaches unity. It is shown that the model of prefractal tube bundles can be used to obtain fitting curves of the permeability of high-porosity metal foams and to provide insight on permeability-porosity correlations of the capillary model of porous media.

Acknowledgments The authors are indebted to anonymous reviewers of Journal of Transport in Porous Media for corrections and suggestions improved this manuscript. 


\section{Appendix}

Regression coefficients of fitting curves in Fig. 2

1) $K_{f}=1.40306 ; K_{*}=56.0927 ; D=1.83418 \mathrm{e}-15 ; R^{2}=0.998324 ; \mathrm{rms}=0.0468576$

2) $K_{f}=2.63448 ; K_{*}=105.217 ; D=1.57839 \mathrm{e}-15 ; R^{2}=0.997981 ; \mathrm{rms}=0.0997096$

3) $p=0.618854 ; r=0.234571 ; R^{2}=0.996432 ; \mathrm{rms}=0.0683661$

4) $\quad p=1.084650 ; r=0.256929 ; R^{2}=0.999578 ; \mathrm{rms}=0.0458130$

\section{References}

Bhattacharya, A., Calmidi, V.V., Mahajan, R.L.: Thermophysical properties of high porosity metal foams. Int. J. Heat Mass Transf. 45, 1017-1031 (2002)

Boomsma, K., Poulikakos, D.: On the effective thermal conductivity of a three-dimensionally structured fluid-saturated metal foam. Int. J. Heat Mass Transf. 44, 827-836 (2001)

Boomsma, K., Poulikakos, D., Ventikos, Y.: Simulations of flow through open cell metal foams using an idealized periodic cell structure. Int. J. Heat Fluid Flow 24, 825-834 (2003a)

Boomsma, K., Poulikakos, D., Zwick, F.: Metal foams as compact high performance heat exchangers. Mech. Mater. 35, 1161-1176 (2003b)

Boyd, D.W.: The sequence of radii of the apollonian packing. Math. Comput. 39, 249-254 (1982)

Costa, A.: Permeability-porosity relationship: a reexamination of the Kozeny-Carman equation based on a fractal pore-space geometry assumption. Geophys. Res. Lett. 33, L02318 (2006)

Deville, S., Saiz, E., Tomsia, A.P.: Freeze casting of hydroxyapatite scaffolds for bone tissue engineering. Biomaterials 27, 5480-5489 (2006)

Donatti, D.A., Vollet, D.R., Mesquita, A.I., Silva, T.F.: Mass fractal characteristics of silica sonogels as determined by small-angle X-ray scattering and nitrogen adsorption. Phys. Rev. B. 71, 014203 (2005)

Fritton, S.P., Weinbaum, S.: Fluid and solute transport in bone: flow-induced mechanotransduction. Ann. Rev. Fluid Mech. 41, 347-374 (2009)

Fuchs, E., Sanden, K.: Prime number and local to global conjectures in Apollonian circle packings (2009) (in press)

Gimenez, D., Perfect, E., Rawls, W.J., Pachepsky, Ya.: Fractal models for predicting soil hydraulic properties: a review. Eng. Geol. 48, 161-183 (1997)

Gurav, J.L., Jung, I., Park H., Kang, E., Nadargi, D.: Silica aerogel: synthesis and applications, J. Nanomater. Article ID 409310 (2010)

Henderson, N., Brettas, J.C., Sacco, W.F.: A three-parameter Kozeny-Carman generalized equation for fractal porous media. Chem. Eng. Sci. 65, 4432-4442 (2010)

Hirata, E., Uo, M., Nodasaka, Y., Takita, H., Ushijima, N., Akasaka, T., Watari, F., Yokoyama, A.: 3D collagen scaffolds coated with multiwalled carbon nanotubes: initial cell attachment to internal surface. J. Biomed. Mater. Res. B Appl. Biomater. 93, B2 (2010)

Jacquenot, G.: 2D Apollonian gasket with four identical circles http://www.mathworks.com/matlabcentral/ fileexchange/15958-2d-apollonian-gasket-with-four-identical-circles (2007)

Jacquin, C.G., Adler, P.M.: Fractal porous media II: geometry of porous geological structures. Transp. Porous Media 2, 571-596 (1987)

Lapidus, L., Pearse, E., Winter S.: Pointwise tube formula for fractal sprays and 2 self-similar tilings with arbitrary generators. arXiv:1006.3807v1 [math.MG] (2010)

Manna, S.S., Herrmann, H.J.: Precise determination of the fractal dimensions of Apollonian packing and space-filling bearings. J. Phys. A Math. Gen. 24, L481-L490 (1991)

Neimark, A.V., Ruetsch, S., Kornev, K.G., Ravikovitch, P.I.: Hierarchical pore structure and wetting properties of single-wall carbon nanotube fibers. Nanoletters 3, 419-423 (2003)

O’Brien, F.J., Harley, B.A., Waller, M.A., Yannas, I.V., Gibson, L.J., Prendergast, P.J.: The effect of pore size on permeability and cell attachment in collagen scaffolds for tissue engineering. Technol. Health Care 15, 3-17 (2007)

Perfect, E., Gentry, R.W., Sukop, M.C., Lawson, J.E.: Multifractal Sierpinski carpets: theory and application to upscaling effective saturated hydraulic conductivity. Geoderma 134, 240-252 (2006)

Perfect, E., Sukop, M.C.: Models relating solute dispersion to pore space geometry in saturated media: a review. Physical and chemical Processes of water and solute transport/retention in soil. Soil Science Society of America: Special publication no. 56, 77-146 (2001) 
Perrier, E., Bird, N., Rieu, M.: Generalizing the fractal model of soil structure, the pore-solid fractal approach. Fractals in Soil Science. Elsevier Science B.V, Amsterdam (2000)

Pierre, A.C., Pajonk, G.M.: Chemistry of aerogels and their Applications. Chem. Rev. 102, 4243-4265 (2002)

Predoi-Racila, M., Stroeb, M.C., Croletb, J.M.: Human cortical bone: the SiNuPrOs model. Part II - a multiscale study of permeability. Comput. Methods Biomech. Biomed. Eng. 13, 81-89 (2010)

Radin, C.: The pinwheel tilings of the plane. Ann. Math. 139, 661-702 (1994)

Rodriguez, E., Giacomelli, F., Vazquez, A.: Permeability-porosity relationship in RTM for different fiberglass and natural reinforcements. J. Compos. Mater. 38, 259-268 (2004)

Shah, R.K.: Laminar flow forced convection in ducts. Academic Press, London (1978)

Thormann, A., Teuscher, N., Pfannmçller, M., Rothe, U., Heilmann, A.: Nanoporous aluminum oxide membranes for filtration and biofunctionalization. Small 3, 1032-1040 (2007)

West, G.B., Brown, J.H., Enquist, B.J.: A general model for the origin of allometric scaling laws in biology. Science 276, 122-126 (1997)

Wolfram S.: Geometry of binomial coefficients http://www.stephen29.com/publications/articles/ca/84geometry/1/text.html (1984)

Yu, B., Liu, W.: Fractal analysis of permeabilities for porous media. Am. Inst. Chem. Eng. J. 50, 46-57 (2004)

Zinovik, I., Poulikakos, D.: On permeability of fractal tube bundles. http://arxiv.org/abs/1109.2302; arXiv:1109.2302 [physics.flu-dyn] (2012) 